\title{
Brentuximab vedotin in combination with rituximab, cyclophosphamide, doxorubicin, and prednisone as frontline treatment for patients with CD30-positive B-cell lymphomas
}

\begin{abstract}
Jakub Svoboda, ${ }^{1}$ Steven M. Bair, ${ }^{1}$ Daniel J. Landsburg, ${ }^{1}$ Sunita Dwivedy Nasta, ${ }^{1}$ Sarah J. Nagle, ${ }^{2}$ Stefan K. Barta, ${ }^{1}$ Nadia Khan, ${ }^{3}$ Joanne Filicko-O'Hara, ${ }^{4}$ Sameh Gaballa, ${ }^{4}$ Lauren Strelec, ${ }^{1}$ Elise Chong, ${ }^{1}$ Sheryl Mitnick, ${ }^{1}$ Terease S. Waite, ${ }^{1}$ Cara King, ${ }_{1}^{1}$ Hatcher Ballard, ${ }^{1}$ Matthew Youngman, ${ }^{1}$ James Gerson, ${ }^{1}$ John P. Plastaras, ${ }^{1}$ Amit Maity, ${ }^{1}$ Agata M. Bogusz, ${ }^{1}$ Stacy S. Hung, ${ }^{5}$ Hisae Nakamura, ${ }^{5}$ Reza Nejati, Christian Steidl, ${ }^{5}$ Megan Lim, ${ }^{1}$ Marco Ruella ${ }^{1}$ and Stephen J. Schuster ${ }^{1}$
\end{abstract}

${ }^{1}$ University of Pennsylvania, Philadelphia, PA, USA; ${ }^{2}$ Oregon Health and Science University, Portland, OR, USA: ${ }^{3}$ Fox Chase Cancer Center, Philadelphia, PA, USA; ${ }^{4}$ Thomas Jefferson University, Philadelphia, PA, USA and ${ }^{5}$ Centre for Lymphoid Cancer, Vancouver, British Columbia, Canada

\section{ABSTRACT}

W e conducted a phase I/II multicenter trial using six cycles of brentuximab vedotin (BV) in combination with rituximab, cyclophosphamide, doxorubicin, and prednisone (R-CHP) for treatment of patients with CD30-positive B-cell lymphomas. Thirty-one patients were evaluable for toxicity and 29 for efficacy including 22 with primary mediastinal B-cell lymphoma, five with diffuse large B-cell lymphoma, and two with gray zone lymphoma. There were no treatmentrelated deaths; $32 \%$ of patients had non-hematologic grade $3 / 4$ toxicities. The overall response rate was $100 \%$ ( $95 \%$ confidence interval $[95 \%$ CI]: 88-100) with $86 \%$ (95\% CI: 68-96) of patients achieving complete response at the end of systemic treatment. Consolidative radiation following end-of-treatment response assessment was permissible and used in $52 \%$ of all patients including $59 \%$ of the patients with primary mediastinal B-cell lymphoma. With a median follow-up of 30 months, the 2 year progression-free survival and overall survival rates were $85 \%(95 \%$ CI: $66-94)$ and $100 \%$, respectively. In the cohort with primary mediastinal B-cell lymphoma, the 2-year progression-free survival rate was $86 \%$ (95\% CI: 62-95). In summary, BV-R-CHP with or without consolidative radiation is a feasible and active frontline regimen for CD30-positive Bcell lymphomas (ClinicalTrials.gov identifier: NCT01994850).

\section{Introduction}

Brentuximab vedotin (BV) is an immunoconjugate consisting of a CD30-directed antibody linked to the anti-microtubule agent auristatin. ${ }^{1} \mathrm{BV}$ is highly active in relapsed and refractory ( $\mathrm{r} / \mathrm{r}$ ) classical Hodgkin lymphoma and in CD30-expressing T-cell lymphomas. ${ }^{2,3}$ In the frontline setting, BV combined with chemotherapy has been recently approved for advanced classical Hodgkin lymphoma and CD30-positive $\left(\mathrm{CD} 30^{+}\right) \mathrm{T}$-cell lymphomas based on results of randomized trials showing benefit of the BV-containing arms. ${ }^{4,5}$

BV targets the cell membrane protein CD30 that is expressed not only by classical Hodgkin lymphoma and some T-cell lymphomas, but at various frequencies also by B-cell non-Hodgkin lymphomas including up to $80 \%$ of primary mediastinal B-cell lymphomas (PMBCL) ${ }^{6.9} \mathrm{PMBCL}$ is a mature large B-cell lymphoma of thymic origin which usually presents with mediastinal masses. It occurs predominantly in young adults and represents about $5 \%$ of aggressive B-cell lymphomas. ${ }^{10}$ While previous classifications considered it as a subtype of diffuse large B-cell lymphoma (DLBCL), PMBCL is now thought of as a distinct clinicopathological entity with clinical features and also a molecular signature that share similarities with
Ferrata Storti Foundation

Haematologica 2021

Volume 106(6):1705-1713

\section{Correspondence:}

JAKUB SVOBODA

jakub.svoboda@pennmedicine.upenn.edu

Received: September 19, 2019.

Accepted: May 14, 2020.

Pre-published: May 15, 2020.

https://doi.org/10.3324/haematol.2019.238675

(C)2021 Ferrata Storti Foundation

Material published in Haematologica is covered by copyright. All rights are reserved to the Ferrata Storti Foundation. Use of published material is allowed under the following terms and conditions:

https://creativecommons.org/licenses/by-nc/4.0/legalcode. Copies of published material are allowed for personal or internal use. Sharing published material for non-commercial purposes is subject to the following conditions:

https://creativecommons.org/licenses/by-nc/4.0/legalcode, sect. 3. Reproducing and sharing published material for commercial purposes is not allowed without permission in writing from the publisher. 
those of classical Hodgkin lymphoma. ${ }^{11}$ Recent efforts using gene expression profiling have aimed at better defining PMBCL at the molecular level and distinguishing it from other aggressive B-cell lymphomas with mediastinal presentation. In particular, the NanoString ${ }^{\odot}$ based Lymph3Cx assay measures expression of 58 genes and allows precise identification of PMBCL cases. ${ }^{12}$

Presently, the optimal frontline management of patients with PMBCL remains controversial. Traditionally, PMBCL was included in clinical trials regarding aggressive B-cell lymphomas and regimens designed for DLBCL were found to be effective. . $^{13-17}$ Rituximab in combination with cyclophosphamide, doxorubicin, vincristine and prednisone (R-CHOP) results in event-free survival rates of about $80 \%$ when followed by consolidative radiation therapy. ${ }^{14,16}$ In 2013, in a phase II trial by Dunleavy et al. including 51 PMBCL patients treated at the National Cancer Institute, dose-adjusted etoposide, prednisone, vincristine, cyclophosphamide, and doxorubicin, plus rituximab (DA-EPOCH-R) without radiotherapy achieved an event-free survival of $93 \% .{ }^{15}$ Many centers in the USA now use this dose-intense DA-EPOCH-R approach for frontline treatment of all PMBCL patients without considering any risk stratification. ${ }^{15}$ Some patients with $\mathrm{r} / \mathrm{r}$ PMBCL, can be salvaged by high-dose chemotherapy with autologous stem cell transplant or radiation, but outcomes tend to be poor. ${ }^{18,19}$ Recently, pembrolizumab and axicabtagene ciloleucel were approved for the treatment of $\mathrm{r} / \mathrm{r}$ PMBCL..$^{20.22}$ While the activity of BV as monotherapy in $\mathrm{r} / \mathrm{r}$ PMBCL has been disappointing, results of a phase II trial using nivolumab in combination with $\mathrm{BV}$ are very encouraging. . $3,24^{24}$

To test the tolerability and make a preliminary assessment of the efficacy of $\mathrm{BV}$ in frontline treatment of B-cell lymphomas, we designed a phase I/II trial using BV in combination with rituximab, cyclophosphamide, doxorubicin, and prednisone (R-CHP) for the treatment of $\mathrm{CD} 30^{+}$ PMBCL, DLBCL, and gray zone lymphoma (GZL) in adult patients (ClinicalTrials.gov identifier: NCT01994850).

\section{Methods}

\section{Study design and patient eligibility}

This multicenter, single arm, phase I/II study enrolled patients aged 18 years and over with untreated histologically confirmed CD30+ PMBCL, DLBCL, or GZL. Patients with any stage, measurable disease, and an Eastern Cooperative Oncology Group Performance Status of 3 or less were eligible. The diagnostic biopsy had to demonstrate at least $1 \%$ or higher expression of CD30 on the lymphoma B cells by immunohistochemistry and was assessed independently by two pathologists. Patients with active central nervous system involvement and uncontrolled systemic infections were excluded. Enrollment began in January 2014 and was completed in April 2017. The primary objective of the phase I portion was to determine the safety of the combination and the maximum tolerated dose of $\mathrm{BV}$ in combination with $\mathrm{R}$-CHP using a de-escalation design. The primary objective of the phase II portion was the overall response rate at the end of systemic treatment as determined by investigator assessment using International Working Group response criteria for non-Hodgkin lymphoma. ${ }^{25}$ Secondary endpoints were 2-year progression-free survival and 2year overall survival for all patients and by each lymphoma subtype (PMBCL, DLBCL, and GZL).

With regard to the toxicity assessment, the study had $90 \%$ power to detect any unforeseen toxicity that occurred in $7 \%$ or
Table 1. The study regimen: brentuximab vedotin in combination with rituximab, cyclophosphamide, doxorubicin, and prednisone (BV-R-CHP).

\begin{tabular}{lcccc} 
Agent & Route & Dose & Cycle 1 & Cycles 2-6 \\
$\begin{array}{l}\text { Prednisone } \\
\text { (or equivalent) }\end{array}$ & $\begin{array}{l}\text { PO } \\
\text { (or IV equivalent) }\end{array}$ & $100 \mathrm{mg}$ & Days 1-5 & Days 1-5 \\
Rituximab & IV & $375 \mathrm{mg} / \mathrm{m}^{2 \dagger}$ & Day 1 and 2 & Day 1 \\
\hline Cyclophosphamide & IV & $750 \mathrm{mg} / \mathrm{m}^{2}$ & Day 2 & Day 1 \\
Doxorubicin & IV & $50 \mathrm{mg} / \mathrm{m}^{2}$ & Day 2 & Day 1 \\
\hline Brentuximab vedotin $^{\ddagger}$ & IV & $1.8 \mathrm{mg} / \mathrm{kg}$ or 1.2 mg/kg & Day 2 & Day 1 \\
\hline
\end{tabular}

Consolidative radiation following the end-of-treatment response assessment was permissible. The rituximab dose was split into $100 \mathrm{mg} / \mathrm{m}^{2}$ and $275 \mathrm{mg} / \mathrm{m}^{2}$ on day 1 and day 2 , respectively of cycle 1. The maximum dose of brentuximab vedotin was $180 \mathrm{mg}$. PO: per os; IV: intravenous.

more of patients. The number of patients required for the trial was determined based on the following assumptions for an optimal two-stage design in order to detect and minimize enrollment if the overall response rate was not greater than $50 \%$ but also to minimize the likelihood of failing to reject the null hypothesis if the overall response rate was at least $70 \%$. Sample size calculations for the stopping rules were based upon a type I error rate of $10 \%$ and type II error rate of $20 \%$. The number of subjects enrolled and evaluable in the phase I cohort was defined as at least six and a maximum of 12 . For the phase II cohort, using the null hypothesis of a $50 \%$ overall response rate, the study required a sample size of 20 patients. Since the phase I subjects were recruited, treated, and followed in the same way as the phase II subjects, the phase I subjects accrued at the phase II dose were included in the efficacy analysis.

The study was conducted in three academic centers in the USA and was run in accordance with the Declaration of Helsinki. Approval from the institutional review board of each center was obtained before initiating the study at each site. All patients signed a written informed consent form before enrollment into the trial.

\section{Treatment protocol and response assessment}

As shown in Table 1, the study treatment protocol consisted of six cycles of $\mathrm{BV}$ administered with the $\mathrm{R}-\mathrm{CHOP}$ regimen without vincristine, including: rituximab $375 \mathrm{mg} / \mathrm{m}^{2}$, cyclophosphamide $750 \mathrm{mg} / \mathrm{m}^{2}$, and doxorubicin $50 \mathrm{mg} / \mathrm{m}^{2}$ on day 1 and prednisone $100 \mathrm{mg}$ (or equivalent) daily on days 1 through 5 of each 21 -day cycle. For cycle 1 , rituximab was split into two doses $\left(100 \mathrm{mg} / \mathrm{m}^{2}\right.$ on day 1 and $275 \mathrm{mg} / \mathrm{m}^{2}$ on day 2) to reduce risks of an infusion reaction to rituximab. We also aimed to separate the initial rituximab infusion from the first exposure to BV to avoid any potential confusion about attribution of infusion reactions. The rest of the agents were given on day 2 (cyclophosphamide, doxorubicin, BV). In cycles 2 through 6 , rituximab was administered at a dose of 375 $\mathrm{mg} / \mathrm{m}^{2}$ on day 1 together with the rest of the agents.

For phase I, the starting dose of BV was $1.8 \mathrm{mg} / \mathrm{kg}$ (maximum dose of $180 \mathrm{mg}$ ) with a $3+3$ de-escalation design to $1.2 \mathrm{mg} / \mathrm{kg}$ (maximum dose of $120 \mathrm{mg}$ ) should dose-limiting toxicities occur during the first 21-day cycle. A dose-limiting toxicity was defined as any grade 3 or 4 non-hematologic toxicity requiring a dose delay over 14 days from the planned day 1 of cycle 2 or any hematologic toxicity not returning to baseline or $\leq$ grade 2 by 21 days from the planned day 1 of cycle 2 . By protocol, at least six patients had to be enrolled and complete one cycle of dosing at the final recommended BV dose in phase I prior to beginning enrollment of patient in phase II. Dose modifications during cycle 2 through 6 for treatment-associated toxicity were specified in the protocol and based on the grade using the Common Terminology Criteria for Adverse Events (CTCAE) version 4.0.

The use of granulocyte-colony stimulating factor (G-CSF) was 


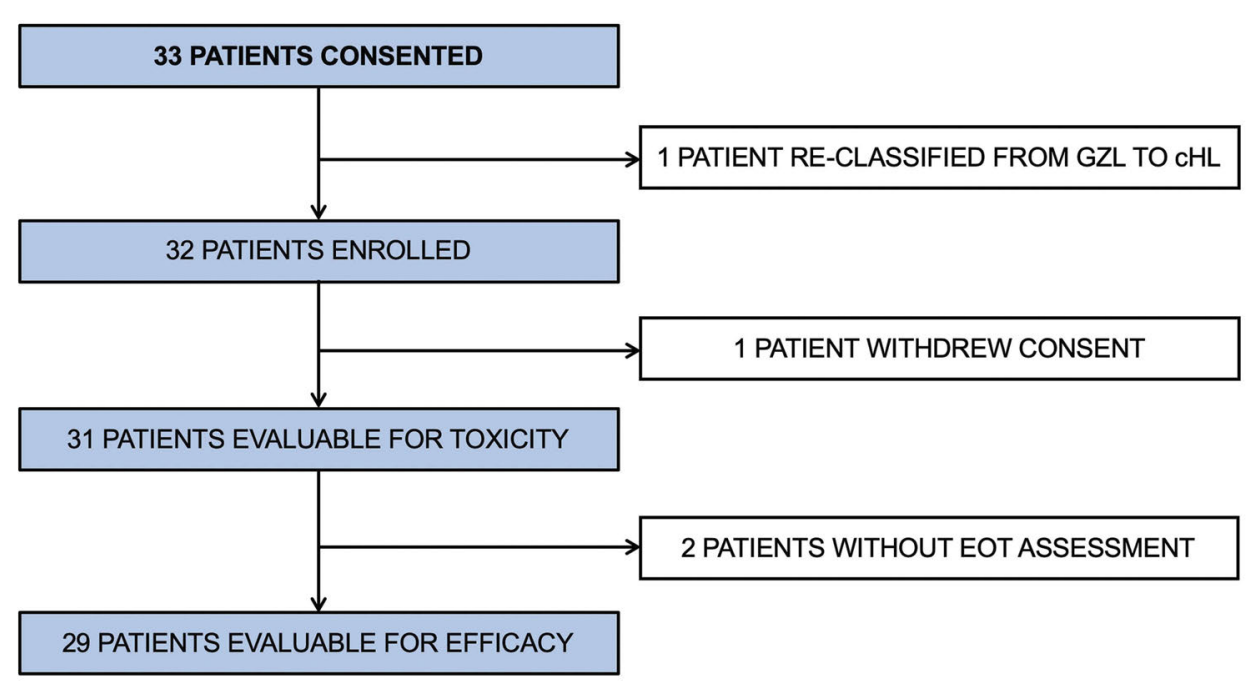

Figure 1. CONSORT diagram. GZL: gray zone lymphoma; $\mathrm{cHL}$ : classical Hodgkin lymphoma; EOT: end of treatment. allowed as per institutional policy. Consolidative radiation therapy was permitted after completion of all systemic therapy and only after end-of-treatment imaging at the discretion of the treating physician.

Treatment response was assessed by imaging with fluorodeoxyglucose-positron emission tomography/computed tomography (FDG-PET/CT) using the revised response criteria for malignant lymphoma described by Cheson et al. ${ }^{25}$ Computed tomography scans were performed after cycles 2 and 4 to monitor for interim response. End-of-treatment imaging was performed 3-5 weeks after completion of systemic therapy using FDG-PET/CT. Consolidative radiation following end-of-treatment response assessment was permissible at the discretion of the treating physician.

\section{Correlative studies}

CD30 expression was determined on the diagnostic tissue biopsies using immunohistochemistry though visual inspection by two independent pathologists. The gene expression analysis (Lymph3Cx) was performed on archival formalin-fixed, paraffinembedded tissue from pre-treatment biopsies. The tissue was examined by a hematopathologist for adequate tumor amount and nucleic acids were extracted from formalin-fixed paraffinembedded scrolls or unstained slides. The Lymph3Cx assay was previously described and validated to aid in the molecular distinction of PMBCL versus DLBCL. ${ }^{26}$ The gene expression assay on the diagnostic tissue was performed in a blinded fashion, and once the assignment of diagnosis by Lymph3Cx was made, a correlation with investigator-based diagnosis (PMBCL vs. DLBCL vs. GZL) was performed.

\section{Statistical analysis}

The overall response rate and complete response rate with a two-sided $95 \%$ exact confidence intervals $(95 \% \mathrm{CI}$ ) were calculated using the Clopper-Pearson method. Two-year progression-free and overall survival rates were estimated using the Kaplan-Meier method. The median follow-up was estimated by the reverse Kaplan-Meier method. ${ }^{27}$ The data cut-off for analysis was January 1, 2019

\section{Results}

\section{Patients' characteristics}

Thirty-three patients gave consent to enrollment in the
Table 2. Patients' characteristics.

\begin{tabular}{lc}
\multicolumn{2}{c}{ All patients $(\mathbf{N}=3$ II) } \\
Age, years & \\
Median & 37 \\
Range & $18-76$ \\
Female & $15(48 \%)$ \\
\hline Elevated LDH & $21(68 \%)$ \\
Stage III-IV & $13(42 \%)$ \\
\hline Lymphoma subtype & \\
PMBL & $23(74 \%)$ \\
DLBCL & $6(19 \%)$ \\
GZL & $2(7 \%)$ \\
ECOG PS & \\
Median & 1 \\
Range & $0-2$ \\
IPI risk category & \\
Low & $15(48 \%)$ \\
Low-intermediate & $11(36 \%)$ \\
High-intermediate & $4(13 \%)$ \\
High & $1(3 \%)$ \\
\hline
\end{tabular}

LDH: lactate dehydrogenase; PMBCL: primary mediastinal B-cell lymphoma; DLBCL: diffuse large B-cell lymphoma; GZL: gray zone lymphoma; ECOG PS: Eastern Cooperative Oncology Group Performance Status; IPI: International Prognostic Index.

trial (Figure 1). One of these patients was subsequently reclassified from having GZL to having classical Hodgkin lymphoma and was taken off the study before starting therapy. Thus, 32 patients were enrolled and received at least one cycle of therapy. One patient withdrew from the study after cycle 1 to receive R-CHOP therapy closer to home. The characteristics of the patients evaluable for toxicity $(n=31)$ are presented in Table 2 . The median age was 37 years (range, 18-76), 50\% of the patients were female, $42 \%$ had stage III/IV disease and $17 \%$ were classified in high-intermediate or high International Prognostic Index (IPI) risk group. ${ }^{28}$ Using traditional clinicopathological criteria, 23 patients had a diagnosis of PMBCL, six were diagnosed as having DLBCL, and two as having GZL. For the PMBCL cohort, $91 \%$ of patients had large mediastinal masses over $7.5 \mathrm{~cm}$ in maximal transverse diameter and $35 \%$ had stage III/IV disease. Two patients were removed from the study (1 patient because of non-compliance and 1 in complete remission because of a regimen violation). Therefore, a total of 29 patients were 
evaluable for efficacy. Of those, 15 patients (52\%) received consolidative radiation after completing BV-R$\mathrm{CHP}$ and final end-of-treatment response assessment. This number included 13 (59\%) of 22 evaluable PMBCL patients. Of those, 8 patients received radiation using protons. Twenty-six patients had archival formalin-fixed paraffin-embedded diagnostic tissue available for Lymph3Cx gene expression analysis.

\section{Safety and feasibility}

Toxicities of this outpatient regimen are listed in Table $3 \mathrm{~A}$ and $\mathrm{B}$. There were no treatment-related or on-study deaths. Using a de-escalation design during the phase I portion of the trial, the first six patients were treated with the initial dose of $1.8 \mathrm{mg} / \mathrm{kg}$ (maximum $180 \mathrm{mg}$ ) in com-

Table 3A. All adverse events at least possibly related to the BV-R-CHP regimen.

\begin{tabular}{|c|c|c|c|c|c|}
\hline All AE & Total $(\%)$ & G1 ( $\%)$ & G2 $(\%)$ & G3 $(\%)$ & G4 $(\%)$ \\
\hline \multicolumn{6}{|l|}{ Hematologic AE } \\
\hline Leukopenia & 77 & 13 & 32 & 16 & 16 \\
\hline Lymphopenia & 84 & 6 & 32 & 23 & 23 \\
\hline Neutropenia & 58 & 3 & 13 & 19 & 23 \\
\hline Febrile neutropenia & 23 & & & 23 & \\
\hline Anemia & 68 & 48 & 16 & 3 & \\
\hline Thrombocytopenia & 23 & 13 & 6 & & 3 \\
\hline \multicolumn{6}{|l|}{ Non hematologic $\mathrm{AE}^{*}$} \\
\hline Abdominal pain & 16 & 10 & 3 & 3 & \\
\hline ALT elevation & 16 & 13 & & 3 & \\
\hline ALKP elevation & 13 & 10 & 3 & & \\
\hline Allergic rhinitis & 13 & 13 & & & \\
\hline Anorexia & 13 & 6 & 6 & & \\
\hline Anxiety & 13 & 13 & & & \\
\hline Arthralgia & 10 & 10 & & & \\
\hline AST elevation & 19 & 19 & & & \\
\hline Chills & 10 & 10 & & & \\
\hline Constipation & 52 & 52 & & & \\
\hline Cough & 19 & 19 & & & \\
\hline Diarrhea & 42 & 29 & 13 & & \\
\hline Dizziness & 29 & 29 & & & \\
\hline Dyspnea & 16 & 16 & & & \\
\hline Fatigue & 65 & 58 & 6 & & \\
\hline Fever & 26 & 19 & 6 & & \\
\hline GERD & 19 & 16 & 3 & & \\
\hline Headache & 35 & 32 & 3 & & \\
\hline Hot flashes & 13 & 13 & & & \\
\hline Hyperglycemia & 16 & 10 & 3 & 3 & \\
\hline Hypoalbuminemia & 10 & 3 & 6 & & \\
\hline Hypocalcemia & 10 & 6 & 3 & & \\
\hline Hypokalemia & 10 & 6 & & & 3 \\
\hline Hyponatremia & 32 & 32 & & & \\
\hline Infusion reaction & 13 & 10 & 3 & & \\
\hline Insomnia & 10 & 10 & & & \\
\hline Motor neuropathy & 10 & 6 & 3 & & \\
\hline Mucositis & 29 & 26 & 3 & & \\
\hline Myalgia & 10 & 10 & & & \\
\hline Nausea & 68 & 52 & 16 & & \\
\hline Pain & 16 & 16 & & & \\
\hline Sensory neuropathy & 61 & 48 & 13 & & \\
\hline Thromboembolic event & 16 & 3 & 6 & 6 & \\
\hline Urinary frequency & 10 & 10 & & & \\
\hline Urinary tract infection & 16 & 9 & 6 & & \\
\hline Vomiting & 13 & 9 & 3 & & \\
\hline
\end{tabular}

bination with standard dose R-CHP with plans to reduce BV to $1.2 \mathrm{mg} / \mathrm{kg}$ (maximum $120 \mathrm{mg}$ ) should there be dose-limiting toxicities. As there were no dose-limiting toxicities during phase I, the BV dose of $1.8 \mathrm{mg} / \mathrm{kg}$ (maximum $180 \mathrm{mg}$ ) was used as the phase II dose. Overall in all patients (combining phase I and phase II cohorts), any grade 3 or 4 toxicity occurred in $84 \%$ of study patients. Hematologic adverse events of any grade were recorded in the majority of patients and in $77 \%$ of patients with grade 3 or 4 toxicities. Of note, $16 \%$ of patients received no G-CSF and 6\% had G-CSF support for only one or two of the six cycles. Non-hematologic grade 3 and 4 toxicities were seen in $32 \%$ of patients, including infections in $15 \%$ of patients (Table 3B). Toxicities occurring in over $10 \%$ of patients included peripheral sensory neuropathy in 19 patients $(61 \%)$ which were either grade $1(48 \%)$ or grade $2(13 \%)$ (Table $3 \mathrm{~A})$. Three patients $(10 \%)$ reported motor neuropathy, two with grade $1(6 \%)$ and one with grade $2(3 \%)$. One patient discontinued protocol treatment after cycle 4 because of sepsis and grade 3 cardiomyopathy. One patient discontinued BV after cycle 5 because of transient grade 2 pneumonitis which was deemed at least possibly related to BV. Only three patients required BV dose reductions to $1.2 \mathrm{mg} / \mathrm{kg}$ because of persistent grade 2 peripheral sensory neuropathy outside of the period of dose-limiting toxicities. In total, two patients enrolled on the study died in the follow-up period. One PMBCL patient developed acute myeloid leukemia 2 years after completion of study treatment and mediastinal radiation therapy and ultimately died of acute myeloid leukemia 39 months after completing study treatment. One patient died of progressive lymphoma 40 months after completing study treatment.

Thromboembolic events were noted in eight patients $(36 \%)$ in the PMBCL cohort. Pulmonary embolism was seen in three patients and upper extremity deep vein thrombosis in five patients. Of those, three events were diagnosed prior to initiating BV-R-CHP and five events were diagnosed while patients were on study treatment. Three of the five patients who had on-treatment events were asymptomatic and thrombosis was reported as an incidental finding on their first computed tomography with intravenous contrast (1 with pulmonary embolism and 1 with internal jugular vein thrombosis). Two of the five patients with on-treatment events had line-associated thromboses.

Table 3B. Grade 3 or 4 adverse events at least possibly related to the BV-R-CHP regimen.

\begin{tabular}{lc}
\hline Grade $3 / 4 \mathrm{AE}$ & $\%$ Total $(n=31)$ \\
\hline All* & 84 \\
Hematologic & 77 \\
Non-hematologic & 32 \\
Infections & 15 \\
Thromboembolic event & 6 \\
ALT elevation & 3 \\
Abdominal pain & 3 \\
LV dysfunction & 3 \\
Hypokalemia & 3 \\
Muscle weakness & 3 \\
Lactic acidosis & 3 \\
Hyperglycemia & 3
\end{tabular}

* Some patients experienced multiple grade $3 / 4$ toxicities so the percentages do not add up. AE: adverse events: ALT: alanine transaminase; LV: left ventricular. 


\section{Efficacy} patients, the overall response rate was 100\% (95\% CI: 88 $100)$ with $86 \%$ (95\% CI: 68-96) of patients achieving a complete response and 14\% (95\% CI: 4-32) achieving a partial response according to FDG-PET/CT imaging at the end of treatment. All four patients with a partial response had a diagnosis of PMBCL and had a low or low-intermediate IPI risk classification. Only two of the four patients with a partial response ultimately progressed. At a median follow-up of 30 months (95\% CI: $26-46$ ), four patients $(14 \%)$ progressed: three with PMBCL and one with GZL. The 2-year progression-free survival rate was $85 \%(95 \%$ CI: 66-94) and the 2-year overall survival was $100 \%$ (Figure 2). Of three patients who were not evaluable per study criteria, two remain progression-free at last followup and the status of one patient is unknown.

In the PMBCL cohort of 22 evaluable patients with a median follow-up of 30 months ( $95 \%$ CI: $23-46$ ), the 2 year progression-free survival rate was $86 \%$ (95\% CI: 62 95) with a 2-year overall survival of $100 \%$ (Figure 2). Of the three PMBCL patients who progressed, two had bulky advanced stage disease with expression of CD30 $\leq 10 \%$ and one had bulky stage I disease with CD30 expression of $1 \%$. There was no statistically significant difference in progression-free survival between the PMBCL patients who received consolidative radiation therapy $(n=13)$ and those who did not $(\mathrm{n}=9)(P=0.95)$.
In the combined phase I/II cohort with 29 evaluable

CD30 expression as determined by immunohistochemistry and response to therapy

While all cases expressed CD30 in at least 1\% of the lymphoma B cells in the tumor biopsy by immunohistochemistry, it was challenging to capture CD30 expression as a single metric since there was great heterogeneity of CD30 expression patterns, as depicted in Figure 3. Additionally, given the $100 \%$ overall response rate and low number of relapses, we could not make any conclusions about correlations between efficacy of the BV-containing regimen and CD30 expression as determined by immunohistochemistry.

\section{Gene expression analysis to improve diagnostic accuracy of primary mediastinal B-cell lymphoma}

Of 29 evaluable patients with CD30 B-cell lymphoma, 26 had a pre-treatment biopsy available (11 excisional biopsies and 15 core needle biopsies). Of the 26 samples, five core needle biopsies did not have adequate tumor content or amounts of extractable RNA for the Lymph3Cx assay. The biopsies of the remaining 21 patients (11 excisional and 10 core needle biopsies) were tested. All three subtypes of $\mathrm{CD} 0^{+} \mathrm{B}$-cell lymphomas as assessed by investigator assessment were tested in blinded fashion by the Lymph3Cx assay and comprised 14 cases of PMBCL, six of DLBCL, and one case of GZL. Of 14 patients with PMBCL by investigator assessment alone, 11 patients $(79 \%)$ had Lymph3Cx probability scores $>0.9$ which were

\section{B Overall Survival (All Patients)}
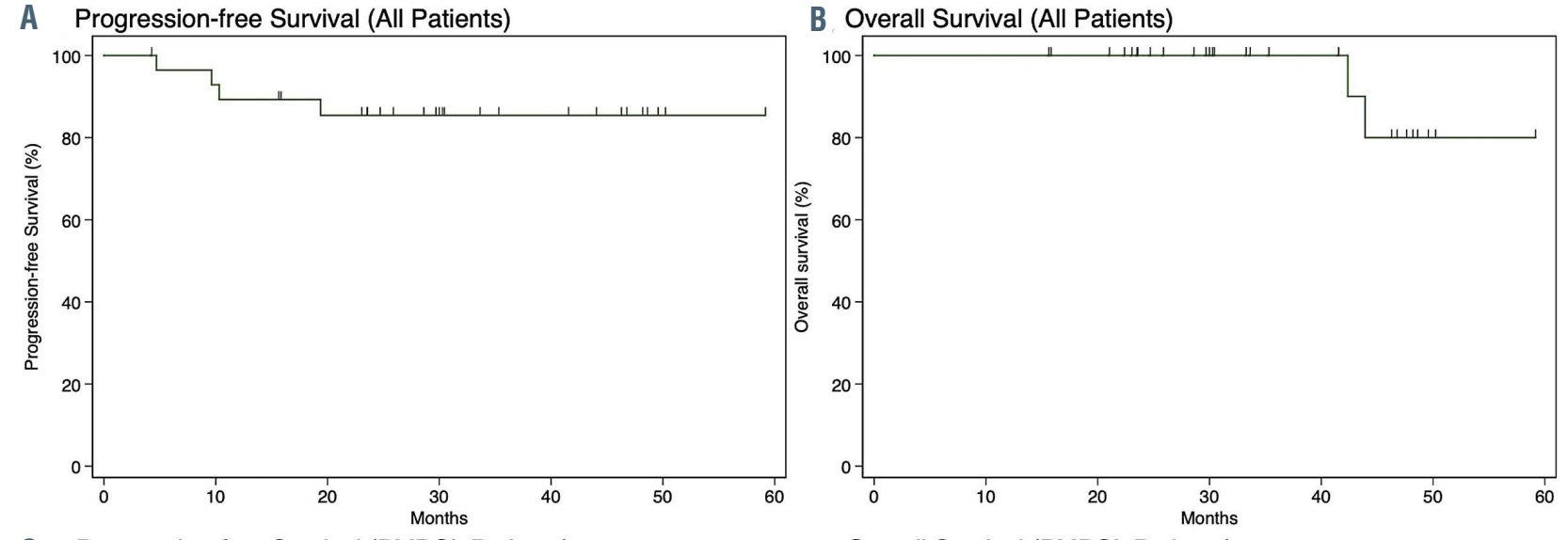

D Overall Survival (PMBCL Patients)

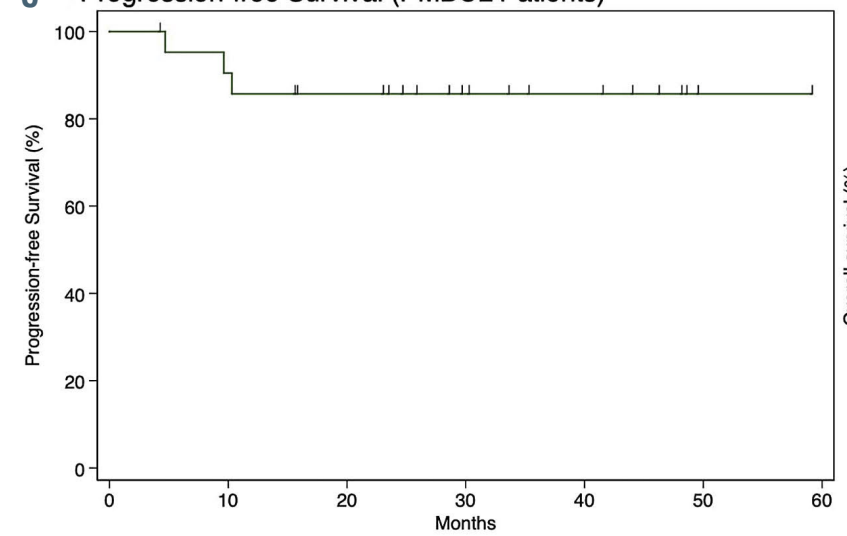

Figure 2. Survival curves for patients who received the BV-R-CHP treatment regimen. (A, B) Progression-free survival (A) and overall survival (B) of all evaluable patients enrolled in the trial $(n=29)$. (C, D) Progression-free survival (C) and overall survival (D) of evaluable patients with primary mediastinal B-cell lymphoma (PMBCL) $(n=22)$. 
CD30

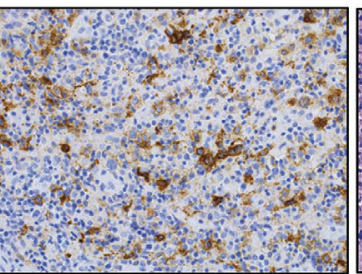

A

CD30 strong vs. dim staining

B

CD30 focal staining

C

CD30 diffuse staining

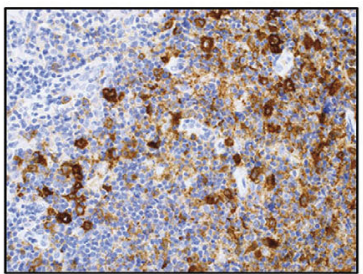

H\&E

CD20
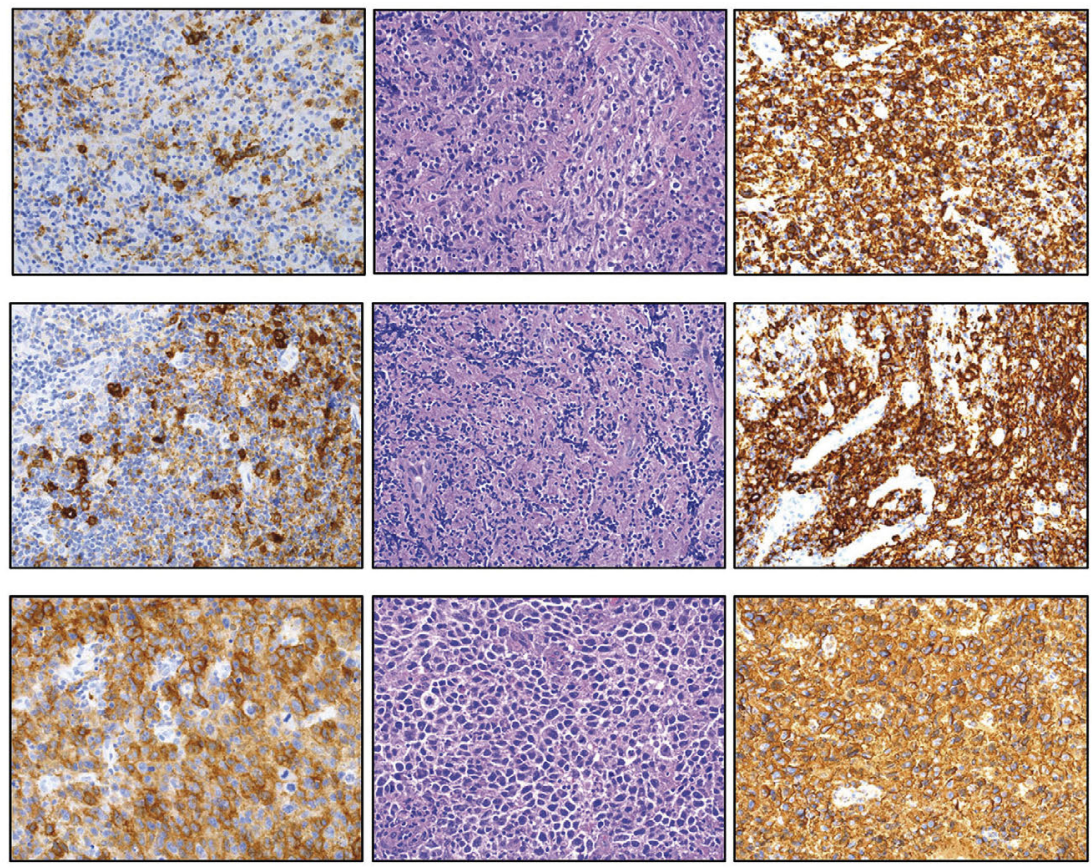

Figure 3. Examples of different CD30 staining patterns by immunohistochemistry in three representative patients with primary mediastinal B-cell lymphoma enrolled on the trial. (A) Heterogeneous staining pattern with strong and dim staining in different areas of the same tumor. (B) Focal staining in one area of the tumor. (C) Diffuse staining throughout the tumor. The antibodies used were CD20 (ready to use, DAKO) and CD30 (ready to use, Leica) and they were detected using a chromogenic substrate, diaminobenzene (Leica). An original magnification x200 was used for all images. H\&E: hematoxylin \& eosin.

consistent with a molecular diagnosis of PMBCL (mPMBCL) by gene expression; two patients scored in the indeterminate category (0.1 to 0.9 ); and one patient scored as having a molecular diagnosis of DLBCL $(<0.1)$ (Figure 4). None of the $\mathrm{CD} 30^{+} \mathrm{B}$-cell lymphoma samples that were felt to be DLBCL by investigator assessment scored as having a molecular diagnosis of PMBCL by Lymph3Cx.

\section{Discussion}

There is a strong rationale for replacing vincristine with $\mathrm{BV}$ in the standard $\mathrm{R}-\mathrm{CHOP}$ regimen for the treatment of $\mathrm{CD}^{+} 0^{+}$aggressive B-cell lymphomas. BV specifically delivers the antimicrotubule agent auristatin to CD30-expressing cells, which could result in improved efficacy from BV and reduced toxicity due to the omission of vincristine. While BV displayed only limited clinical activity as monotherapy in aggressive $\mathrm{r} / \mathrm{r}$ B-cell lymphomas, it has not been widely studied in the frontline setting or in combination with chemotherapy. ${ }^{24,29}$ In our phase I/II study, we showed that a frontline regimen using BV at a dose of $1.8 \mathrm{mg} / \mathrm{kg}$ in combination with R-CHP for patients with $\mathrm{CD} 30^{+} \mathrm{B}$-cell lymphomas has an acceptable toxicity profile and is highly active.

Our study included a heterogeneous group of B-cell lymphomas, but the majority of the patients had a clinicopathological diagnosis of PMBCL. For many reasons, this is a challenging population to study in a frontline setting. PMBCL is a rare and clinically heterogeneous lymphoma. Patients with this type of lymphoma often present with an acute onset of pulmonary symptoms necessitating urgent therapy which may lead to a selection bias in non- randomized studies. While several frontline treatment approaches are effective in $\mathrm{PMBCL}$, there are unique challenges in this population of patients. DA-EPOCH-R is a highly active dose-intense regimen, but it requires central venous access, use of growth factors, frequent blood testing, and inpatient admission at some institutions. RCHOP is easier to administer, but the excellent outcomes in PMBCL are achieved using consolidative radiation therapy, which may cause long-term toxicities. ${ }^{14,16,30,31}$ While a recently published phase III trial comparing $\mathrm{R}-\mathrm{CHOP}$ versus DA-EPOCH-R in DLBCL included a small cohort of PMBCL cases $(n=35)$, it was not statistically powered to detect the differences in this lymphoma subtype. ${ }^{17}$

We recognize that it is difficult to compare regimens across different trials, but outcomes within the PMBCL cohort in our study are comparable to previously published results for patients treated with R-CHOP with radiotherapy or dose-intense regimens such as DAEPOCH-R. ${ }^{14-16,19,30,32}$ For lymphoma subtypes other than $\mathrm{PMBCL}$, the numbers of patients were too small to make any efficacy conclusions regarding BV-R-CHP. One of two patients with GZL relapsed after achieving a complete response and none of the five patients with CD30+ DLBCL relapsed, which is encouraging. Interestingly, preliminary results from another phase II trial (ClinicalTrial.gov identifier: NCT01925612) using BV-R-CHOP in DLBCL (without any requirement for $\mathrm{CD} 30$ staining) documented an overall response rate of $97 \%$ in the initial 30 evaluable patients. None of the CD $30^{+}$DLBCL patients in the preliminary report relapsed, but the median follow-up of 5 months was short. 3

Regarding toxicity of the BV-R-CHP regimen, there were no study-related deaths. With the caveats about 


\section{Color Key}

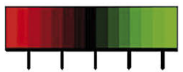

\section{$-2 \quad 0 \quad 2$}

Row Z-Score

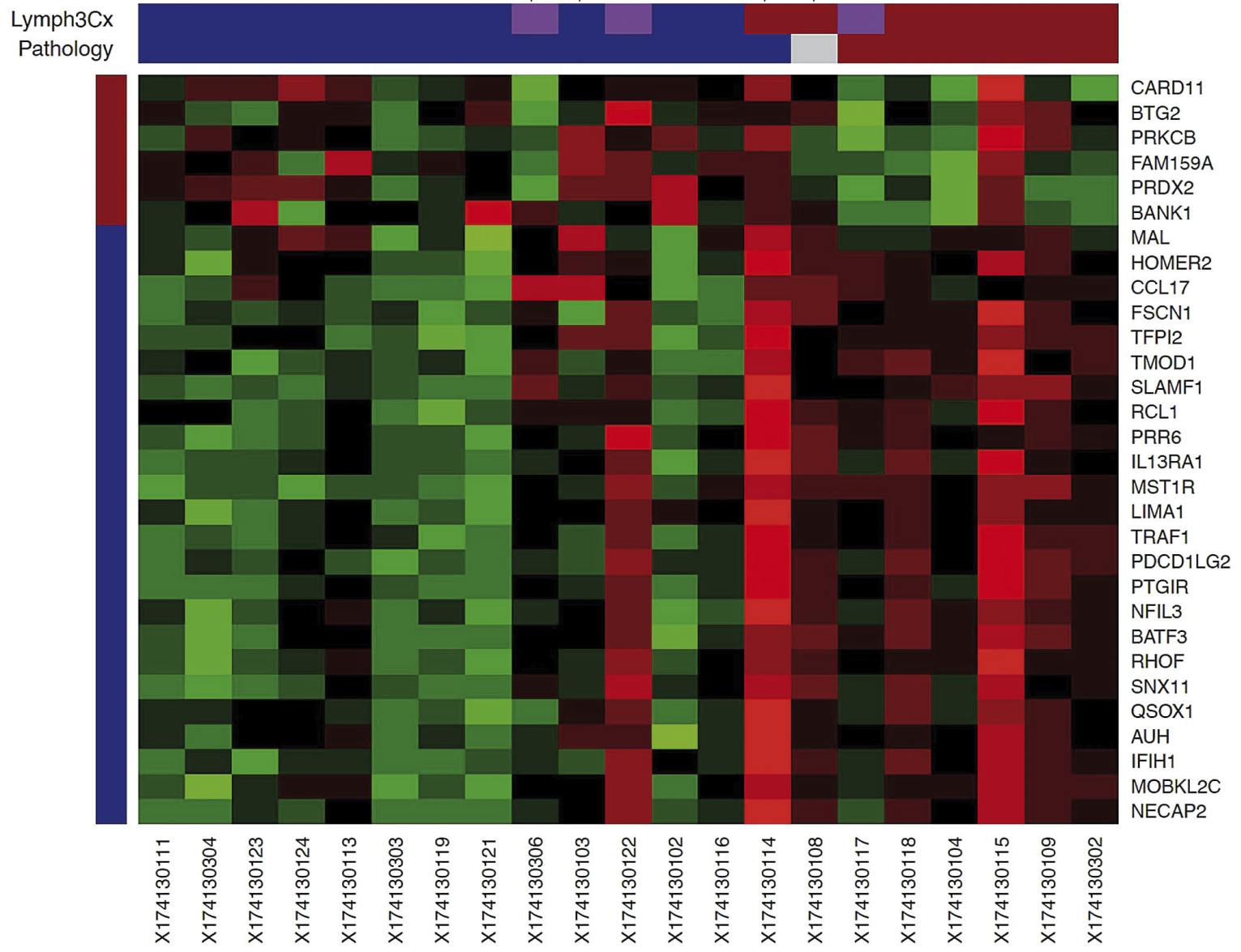

Figure 4. Correlation of Lymph3Cx results with standard clinicopathological diagnoses made by the investigators. DLBCL: diffuse large B-cell lymphoma; GZL: gray zone lymphoma; PMBCL: primary mediastinal B-cell lymphoma. Patients who progressed are labeled by an arrow. Of 14 patients with PMBCL diagnosed by investigator assessment alone, 11 patients $(79 \%)$ had Lymph3Cx probability scores $>0.9$, which were consistent with a molecular diagnosis of PMBCL by gene expression analysis, two patients (14\%) scored in the indeterminate category ( 0.1 to 0.9$)$, and one patient ( $7 \%)$ scored as having DLBCL (< 0.1$)$

cross-trial comparisons of studies, the rate of grade 3 or 4 hematologic and non-hematologic toxicities was similar or lower compared to the rates reported for R-CHOP. ${ }^{17,34}$ When compared to the DA-EPOCH-R arm from the recently published randomized trial in DLBCL, there appears to be less toxicity with BV-R-CHP in our study. ${ }^{17}$ However, one limitation of this comparison is the younger median age of patients in our cohort. Neuropathy is of particular concern with a BV-containing regimen and was closely monitored in our study. While peripheral sensory neuropathy was reported in $61 \%$ of patients, no patient experienced grade 3 or 4 neuropathy. This lack of severe peripheral neuropathy may again relate to the young age of our patients and the fact that our BV-containing regimen did not contain additional vinca alkaloids, in contrast to some of the other BV-containing combinations used frontline. ${ }^{4,33}$ There were no unexpected opportunistic infections using the combination of rituximab and BV. The administration of G-CSF was not consistent across the participating institutions in our study, but over $20 \%$ of patients did not require G-CSF at all or its use was limited to one or two cycles. However, considering that $23 \%$ of patients experienced febrile neutropenia, empiric use of G-CSF should be considered in patients being treated with BV-R-CHP. With regard to long-term toxicities, one patient developed acute myeloid leukemia 2 years after completing the trial therapy and radiation. It is not possible to determine the association between the protocol treatment and her leukemia, but the fact that the patient's mother died of acute myeloid leukemia and the patient had normal cytogenetics (rare in secondary leukemias) is suggestive of other contributing factors.

The high rate of thrombosis in the PMBCL cohort is of special interest. Thromboses were found in over one third of PMBCL patients and approximately $50 \%$ were diagnosed prior to initiation of therapy. This high risk of thrombosis in PMBCL patients was described with similar frequency in retrospective studies and is not likely to be related to BV-R-CHP. . $^{1935}$ This finding warrants further investigations about screening, the potential contribution 
of central lines to thrombosis, and any possible role for prophylactic anticoagulation in PMBCL patients.

We also attempted to define clinical and pathological factors which would correlate with outcomes of patients receiving BV-R-CHP therapy for $\mathrm{CD} 30^{+} \mathrm{B}$-cell lymphomas. IPI risk group, which is well-established as a prognostic factor for outcomes of frontline treatments in DLBCL, did not clearly correlate with complete response rate, progression-free survival or overall survival in our study. This could be due to the small number of patients in the high or high-intermediate IPI risk category. Furthermore, the majority of our patients had PMBCL and the utility of the IPI has some limitations as most patients are young and present with limited stage disease. For our ancillary studies, we planned an analysis of CD30 expression by immunohistochemistry and correlation with outcomes as there is controversy over the impact of CD30 status on the efficacy of BV ${ }^{3,3,5,37}$ However, this proved difficult because of the very low number of relapses and heterogeneity of CD30 staining patterns in neoplastic cells (Figure 3). Additional studies beyond a simple determination of the percentage of $\mathrm{CD} 30^{+}$cells by immunohistochemistry and visual assessment will need to be applied and other groups have attempted this with some success. ${ }^{37,38}$

Among 21 patients who had pre-treatment tissue analyzed by LymphC3x, we found that there was discordance between the protocol-specified standard clinicopathological diagnosis of PMBCL and the gene expression-based method. These findings are thought-provoking since, in small trials of PMBCL, even a few misclassified patients may have a great impact on interpretation of the results. We believe that developing objective diagnostic criteria based on quantitative methods, such as gene expression signatures, will be an important step in designing treatment strategies for B-cell lymphoma patients with mediastinal lesions and for comparing results across PMBCL trials.

This trial is limited by the small number of evaluable patients and diagnostic heterogeneity. However, the entities included are rare, and we involved three institutions to enroll 32 patients. One of the challenges when interpreting the clinical efficacy and progression-free/overall survival data of patients treated with the BV-R-CHP regimen is the fact that consolidative radiation was used in about $50 \%$ of all patients enrolled on this trial. The protocol was designed in 2011-2012 when R-CHOP followed by consolidative radiation therapy was utilized by most centers for PMBCL patients. Therefore, the protocol allowed investigators to use consolidative radiotherapy after completion of BV-R-CHP. It is important to note that the end-of-treatment response assessment was performed before radiation. Interestingly, there were no statistically significant differences in progression-free or overall survival between patients who received consolidative radiation and those who did not. There were no clear differences in patients' characteristics between those who received consolidative radiotherapy and those who did not other than institutional practice differences. Of the four patients who did not achieve metabolic complete response on end-of-treatment imaging, two received consolidative radiation therapy and two did not. Longer follow-up will be necessary to determine whether there are any long-term toxicities of radiation in the study participants (with the majority of patients having received proton radiation). Of note, an ongoing randomized trial in patients with PMBCL may allow us to determine whether consolidative radiation therapy after frontline chemoimmunotherapy is necessary in patients who achieve metabolic complete response after systemic treatment (ClinicalTrial.gov identifier: NCT01599559).

$\mathrm{BV}$ in combination with R-CHP with or without consolidative radiation therapy is a feasible and active frontline treatment in patients with $\mathrm{CD} 30^{+} \mathrm{B}$-cell lymphomas. The safety profile of this regimen, ease of administration and preliminary efficacy data appear promising. The next generation of trials in $\mathrm{CD} 30^{+} \mathrm{B}$-cell lymphomas and PMBCL should take into consideration the clinical and biological heterogeneity of these lymphomas. Ultimately, developing treatment regimens that will be tailored to unique tumor and patient characteristics will result in improved outcomes and will minimize treatment-related toxicities.

\section{Disclosures}

JS has received research funding from Seattle Genetics, BMS, Merck, Celgene, Incyte, and Pharmacyclics and honoraria for consultancy from Seattle Genetics, BMS, Kite, Kyowa, and Astra-Zeneca. DL has received research funding from Curis, Takeda, and Triphase and honoraria for consultancy from Curis and Celgene and for participation in a speakers' bureau from Seattle Genetics. SDN has received research funding from Roche, Incyte, Rafael, Aileron, Takeda/Millenium, Debiopharm, and Atara and honoraria for consultancy from Merck. SKB has received research funding from Seattle Genetics, Merck, Celgene, Takeda, and Bayer, and honoraria for consultancy from Janssen, for educational activity from Mundipharma, and for advisory board work from Seattle Genetics. NK has received research funding from BMS and Janssen and honoraria for consultancy from Seattle Genetics and for participation in a speakers' bureau from Genentech. EAC has received honoraria for consultancy from Novarti. JG has received honoraria for consultancy from Seattle Genetics. CS has received research funding from $B M S$ and Tioma and honoraria for consultancy from Seattle Genetics, Roche, Bayer, and Curis; $C S$ is also named as an inventor on a patent filed by the National Cancer Institute "Methods for determining lymphoma type". SJS has received research funding from Celgene, Genentech, Merck, and Novartis and honoraria for consultancy from Celgene, Dava Oncology, Merck, Nordic Nanovector, Novartis, Genentech, Gilead, and Pfizer. AM has received research funding from Merck. MSL has received honoraria for consultancy from Seattle Genetics. SMB, SJN, JEF, SG, LS, $S M, T S W, C K, H J B, M Y, J P P, A M B, S S H, H K, R N$, and $M R$ have no conflicts of interest to disclose.

\section{Contributions}

JS and SJS designed the study; JS analyzed the data and wrote the manuscript; $S M B, S J N, L S, H J B, M Y$ and $S M$ performed research and analyzed the data; $D L, S D N, S K B, N K$, $J E F, S G, E A C, J G, T S W, C K, J P P$ and $A M$ provided treatment and follow-up for patients on the study; $A M B, S S H, R N, C S$, $M S L$ and MR were involved in research design and analysis of the correlative studies. All authors read, critically reviewed and approved the manuscript. The authors would like to thank Dr. Elena Gitelson, in memoriam, who was involved with the initial trial conception and protocol discussions. 


\section{References}

1. Younes A, Yasothan U, Kirkpatrick P. Brentuximab vedotin. Nat Rev Drug Discov. 2012;11:19.

2. Younes A, Gopal AK, Smith SE, et al. Results of a pivotal phase II study of brentuximab vedotin for patients with relapsed or refractory Hodgkin's lymphoma. J Clin Oncol. 2012;30(18):2183-2189.

3. Pro B, Advani R, Brice P, et al. Brentuximab vedotin (SGN-35) in patients with relapsed or refractory systemic anaplastic large-cell lymphoma: results of a phase II study. J Clin Oncol. 2012;30(18):2190-2196.

4. Connors JM, Jurczak W, Straus DJ, et al. Brentuximab vedotin with chemotherapy for stage III or IV Hodgkin's lymphoma. N Engl J Med. 2018;378(4):331-344

5. Horwitz S, O'Connor OA, Pro B, et al. Brentuximab vedotin with chemotherapy for CD30-positive peripheral T-cell lymphoma (ECHELON-2): a global, doubleblind, randomised, phase 3 trial. Lancet. 2019;393(10168):229-240.

6. Hu S, Xu-Monette ZY, Balasubramanyam A, et al. CD30 expression defines a novel subgroup of diffuse large B-cell lymphoma with favorable prognosis and distinct gene expression signature: a report from the International DLBCL Rituximab-CHOP Consortium Program Study. Blood. 2013;121(14):2715-2724.

7. Lu T-X, Liang J-H, Miao Y, et al. Epstein-Barr virus positive diffuse large B-cell lymphoma predict poor outcome, regardless of the age. Sci Rep. 2015;5:12168.

8. Slack GW, Steidl C, Sehn LH, Gascoyne RD. CD30 expression in de novo diffuse large B-cell lymphoma: a population-based study from British Columbia. Br J Haematol. 2014;167(5):608-617.

9. Higgins JP, Warnke RA. CD30 expression is common in mediastinal large B-cell lymphoma. Am J Clin Pathol. 1999;112(2):241247.

10. Barth TF, Leithauser F, Joos S, Bentz M, Moller P. Mediastinal (thymic) large B-cell lymphoma: where do we stand? Lancet Oncol. 2002;3(4):229-234.

11. Rosenwald A, Wright G, Leroy K, et al. Molecular diagnosis of primary mediastinal $\mathrm{B}$ cell lymphoma identifies a clinically favorable subgroup of diffuse large B cell lymphoma related to Hodgkin lymphoma. J Exp Med. 2003;198(6):851-862

12. Mottok A. Steidl C. Biology of classical Hodgkin lymphoma: implications for prognosis and novel therapies. Blood. 2018;131 (15):1654-1665

13. Zinzani PL, Stefoni V, Finolezzi E, et al. Rituximab combined with MACOP-B or VACOP-B and radiation therapy in primary mediastinal large B-cell lymphoma: a retrospective study. Clin Lymphoma Myeloma. 2009;9(5):381-385
14. Rieger M, Österborg A, Pettengell R, et al. Primary mediastinal B-cell lymphoma treated with CHOP-like chemotherapy with or without rituximab: results of the Mabthera International Trial Group study. Ann Oncol. 2010;22(3):664-670.

15. Dunleavy K, Pittaluga S, Maeda LS, et al. Dose-adjusted EPOCH-rituximab therapy in primary mediastinal B-cell lymphoma. N Engl J Med. 2013;368(15):1408-1416.

16. Gleeson M, Hawkes EA, Cunningham D, et al. Rituximab, cyclophosphamide, doxorubicin, vincristine and prednisolone ( $\mathrm{R}$ $\mathrm{CHOP}$ ) in the management of primary mediastinal B-cell lymphoma: a subgroup analysis of the UK NCRI R-CHOP 14 versus 21 trial. Br J Haematol. 2016;175(4):668-672.

17. Bartlett NL, Wilson WH, Jung S-H, et al. Dose-adjusted EPOCH-R compared with R$\mathrm{CHOP}$ as frontline therapy for diffuse large B-cell lymphoma: clinical outcomes of the phase III Intergroup Trial Alliance/CALGB 50303. J Clin Oncol;2019; 37(21):1790-1799.

18. Kuruvilla J, Pintilie M, Tsang R, Nagy T, Keating A, Crump M. Salvage chemotherapy and autologous stem cell transplantation are inferior for relapsed or refractory primary mediastinal large B-cell lymphoma compared with diffuse large B-cell lymphoma. Leuk Lymphoma. 2008;49(7):1329-1336.

19. Giulino-Roth L, O'Donohue T, Chen Z, et al. Outcomes of adults and children with primary mediastinal B-cell lymphoma treated with dose-adjusted EPOCH-R. Br J Haematol. 2017;179(5):739-747

20. Armand P, Rodig SJ, Melnichenko V, et al. Pembrolizumab in patients with relapsed or refractory primary mediastinal large B-cell lymphoma (PMBCL): data from the Keynote-013 and Keynote-170 studies. Blood. 2018;132(Suppl 1):228.

21. Neelapu SS, Locke FL, Bartlett NL, et al Axicabtagene ciloleucel CAR T-cell therapy in refractory large B-cell lymphoma. N Engl J Med. 2017;377(26):2531-2544.

22. Zinzani PL, Ribrag V, Moskowitz CH, et al. Safety and tolerability of pembrolizumab in patients with relapsed/refractory primary mediastinal large B-cell lymphoma. Blood. 2017;130(3):267-270

23. Moskowitz AJ, Santoro A, Gritti G, et al. Nivolumab combined with brentuximab vedotin for relapsed/refractory primary mediastinal large B-cell lymphoma: preliminary results from the phase 2 CheckMate 436 trial. Blood. 2018;132(Suppl 1):1691.

24. Zinzani PL, Pellegrini C, Chiappella A, et al. Brentuximab vedotin in relapsed primary mediastinal large B-cell lymphoma: results from a phase 2 clinical trial. Blood. 2017;129(16):2328-2330.

25. Cheson BD, Pfistner B, Juweid ME, et al. Revised response criteria for malignant lymphoma. J Clin Oncol. 2007;25(5):579-586

26. Mottok A, Wright G, Rosenwald A, et al. Molecular classification of primary mediastinal large B-cell lymphoma using routinely available tissue specimens. Blood. 2018;132 (22):2401-2405

27. Schemper M, Smith TL. A note on quantifying follow-up in studies of failure time. Control Clin Trials. 1996;17(4):343-346.

28. International Non-Hodgkin's Lymphoma Prognostic Factors Project. A predictive model for aggressive non-Hodgkin's lymphoma. N Engl J Med. 1993;329(14):987994

29. Jacobsen ED, Sharman JP, Oki Y, et al. Brentuximab vedotin demonstrates objective responses in a phase 2 study of relapsed/refractory DLBCL with variable CD30 expression. Blood. 2015;125(9):1394 1402.

30. Shah NN, Szabo A, Huntington SF, et al. RCHOP versus dose-adjusted R-EPOCH in frontline management of primary mediastinal B-cell lymphoma: a multi-centre analysis. Br J Haematol. 2018;180(4):534-544.

31. Soumerai JD, Hellmann MD, Feng Y, Sohani AR, Toomey CE, Barnes JA. Treatment of primary mediastinal B-cell lymphoma with rituximab, cyclophosphamide, doxorubicin, vincristine and prednisone is associated with a high rate of primary refractory disease. Leuk Lymphoma. 2014;55(3):538-543.

32. Martelli M, Ceriani L, Zucca E, et al. $[18 F]$ fluorodeoxyglucose positron emission tomography predicts survival after chemoimmunotherapy for primary mediastinal large B-cell lymphoma: results of the International Extranodal Lymphoma Study Group IELSG-26 study. J Clin Oncol 2014;32(17):1769-1775.

33. Bartlett NL, Farber CM, Yasenchak CA, et al. Updated results of a phase II trial of brentuximab vedotin combined with R-CHOP in frontline treatment of patients (pts) with high-intermediate/high-risk diffuse large Bcell lymphoma (DLBCL). J Clin Oncol. 2015:33(15_suppl):8506.

34. Vitolo $U$, Trněný $M$, Belada $D$, et al. Obinutuzumab or rituximab plus cyclophosphamide, doxorubicin, vincristine, and prednisone in previously untreated diffuse large B-cell lymphoma. J Clin Oncol. 2017:35(31):3529-3537.

35. Lekovic D, Miljic P, Mihaljevic B. Increased risk of venous thromboembolism in patients with primary mediastinal large B-cell lymphoma. Thromb Res. 2010;126(6): 477-480.

36. Duvic M, Tetzlaff MT, Gangar P, Clos AL Sui D, Talpur R. Results of a phase II trial of brentuximab vedotin for CD30+ cutaneous T-cell lymphoma and lymphomatoid papulosis. J Clin Oncol. 2015;33(32):3759-3765.

37. Kim YH, Prince HM, Whittaker S, et al Outcomes by CD30 expression in patients with CTCL receiving brentuximab vedotin (BV) vs physician's choice (PC) in the phase 3 ALCANZA study. J Clin Oncol. 2017:35(15 suppl):7517.

38. Sabattini E, Pizzi M, Tabanelli V, et al. CD30 expression in peripheral T-cell lymphomas. Haematologica. 2013;98(8):e81-e82. 